\title{
(6) OPEN ACCESS \\ Modelling attending physician productivity in the emergency department: a multicentre study
}

\author{
Joshua W Joseph, ${ }^{1,2}$ Samuel Davis, ${ }^{3}$ Elissa H Wilker, ${ }^{2,4,5}$ Matthew L Wong, ${ }^{1,2}$ \\ Ori Litvak, ${ }^{3}$ Stephen J Traub, ${ }^{6}$ Larry A Nathanson, ${ }^{1,2}$ Leon D Sanchez ${ }^{1,2}$
}

'Department of Emergency Medicine, Beth Israel Deaconess Medical Center, Boston,

Massachusetts, USA

${ }^{2}$ Harvard Medical School,

Boston, Massachusetts, USA

${ }^{3}$ LogixHealth, Bedford,

Massachusetts, USA

${ }^{4}$ Cardiovascular Epidemiology Research Unit, Division of

Cardiovascular Medicine, Beth Israel Deaconess Medical

Center, Boston, Massachusetts, USA

${ }^{5}$ Department of Epidemiology, Harvard School of Public Health, Boston, Massachusetts, USA ${ }^{6}$ Department of Emergency Medicine, Mayo Clinic Arizona, Scottsdale, Arizona, USA

\section{Correspondence to}

Dr Joshua W Joseph, Department of Emergency

Medicine, Beth Israel Deaconess Medical Center, 1 Deaconess Road, Boston, MA 02215, USA; jwjoseph@bidmc.harvard.edu

Received 18 September 2017 Revised 13 February 2018

Accepted 19 February 2018

Published Online First

15 March 2018

\section{ABSTRACT \\ Objectives Emergency physician productivity, often} defined as new patients evaluated per hour, is essential to planning clinical operations. Prior research in this area considered this a static quantity; however, our group's study of resident physicians demonstrated significant decreases in hourly productivity throughout shifts. We now examine attending physicians' productivity to determine if it is also dynamic.

Methods This is a retrospective cohort study, conducted from 2014 to 2016 across three community hospitals in the north-eastern USA, with different schedules and coverage. Timestamps of all patient encounters were automatically logged by the sites' electronic health record. Generalised estimating equations were constructed to predict productivity in terms of new patients per shift hour.

Results 207169 patients were seen by 64 physicians over 2 years, comprising 9822 physician shifts. Physicians saw an average of 15.0 (SD 4.7), 20.9 (SD 6.4) and 13.2 (SD 3.8) patients per shift at the three sites, with 2.97 (SD 0.22), 2.95 (SD 0.24) and 2.17 (SD 0.09) in the first hour. Across all sites, physicians saw significantly fewer new patients after the first hour, with more gradual decreases subsequently. Additional patient arrivals were associated with greater productivity; however, this attenuates substantially late in the shift. The presence of other physicians was also associated with slightly decreased productivity.

Conclusions Physician productivity over a single shift follows a predictable pattern that decreases significantly on an hourly basis, even if there are new patients to be seen. Estimating productivity as a simple average substantially underestimates physicians' capacity early in a shift and overestimates it later. This pattern of productivity should be factored into hospitals' staffing plans, with shifts aligned to start with the greatest volumes of patient arrivals.

\section{INTRODUCTION}

Physician productivity, defined as new patients evaluated per hour, is an essential ED operations metric. The existing ED operations literature has generally defined this as an average (new patients per hour) calculated over an entire shift. ${ }^{12}$ Our recent study of resident physicians' productivity showed that the number of new patients a resident sees declines significantly over subsequent hours of the shift. ${ }^{3}$ Correspondingly, an average measurement of productivity will tend to overestimate a physician's capacity to see patients late in the shift and underestimate their potential at the beginning.

\section{Key messages}

What is already known on this subject

- The productivity of individual emergency physicians is reported in terms of an average number of new patients seen per hour (or a derived measure), calculated across an entire shift. A previous study of resident (trainee) emergency physicians demonstrated that residents typically do not see a steady number of new patients per hour, they see fewer patients in each consecutive hour of their shift.

\section{What this study adds}

- In this retrospective study of three community hospital EDs with varying shift lengths, emergency physicians evaluated significantly more new patients early in a shift, and few at the end of a shift, rather than at a steady pace. Physicians were also less responsive to the arrival of new patients later in the shift, suggesting that the beginning of physicians' shifts should be aligned with periods of highest volume, and staggered to ensure that there is an adequate number of patients to be seen early in the shift.

Anticipating how many patients a physician sees during the course of a shift, and when in the shift the physician sees them, are two key factors to consider in staffing an ED appropriately and have important implications. While there are many factors governing patient throughput, the doorto-doctor time (an important clinical measure and a quality metric mandated for many hospitals) depends on the presence of a physician available to see new patients. ${ }^{45}$ If physician productivity is dynamic, it would be essential to align physicians' periods of greatest capacity to see new patients with patient arrivals. An accurate picture of ED physicians' work habits is also a prerequisite to investigating how many patients a physician can be expected to see safely during a shift and how many physicians are needed to efficiently staff a particular ED.

In this study, we examined the number of new patients that attending physicians evaluated during each hour throughout the course of 8-hour and 9-hour shifts to determine if productivity stayed constant or if it changed by shift hour. 
Table 1 Characteristics of the study participants and sites evaluated

\begin{tabular}{|c|c|c|c|}
\hline Characteristics & Site 1 & Site 2 & Site 3 \\
\hline Yearly visits & 46000 & 32000 & 28000 \\
\hline Shifts per day & $6-7$ & 4 & $3-4$ \\
\hline Shifts evaluated & 4444 & 2920 & 2458 \\
\hline $\begin{array}{l}\text { Physicians working during the } \\
\text { study period }\end{array}$ & 22 & 17 & 25 \\
\hline $\begin{array}{l}\text { Maximum number of } \\
\text { concurrent physicians }\end{array}$ & 3 & 2 & 2 \\
\hline $\begin{array}{l}\text { Hours of single provider } \\
\text { coverage }\end{array}$ & 6 & 12 & 12 \\
\hline $\begin{array}{l}\text { Hours of mid-level provider } \\
\text { coverage }\end{array}$ & 16 & 18 & 14 \\
\hline Mean patients per shift & 15.0 (SD 4.7) & 20.9 (SD 6.4) & 13.2 (SD 3.8) \\
\hline $\begin{array}{l}\text { Percentage Emergency Severity } \\
\text { Index } 3 \text { or more acute }\end{array}$ & 73.1 & 76.2 & 76.9 \\
\hline
\end{tabular}

\section{METHODS}

\section{Study design}

This was a retrospective, observational study of deidentified timestamp data.

\section{Study setting}

The study setting was three separate suburban community hospital EDs in north-eastern USA. The three sites have different schedules and amounts of overlapping coverage, but have similarly structured 8-hour and 9-hour shifts. Physicians assign themselves to patients at all sites ad libitum once patients have entered the department. The largest site (site 1) has 46000 visits per year, with six to seven overlapping daily physician shifts based on anticipated demand; the middle site (site 2) has 32000 visits per year, with four physician shifts; and the lowest volume site (site 3) has 28000 visits per year, with three to four physician shifts. Additional characteristics of the sites are described in table 1 . Nine-hour shifts at all sites have the final hour of the shift dedicated to shift wrap-up, during which physicians are not expected to see new patients and have the expectation that all signed-out patients have a set plan for their disposition.

Sites 1 and 3 have dedicated fast-track areas, limited to patients with Emergency Severity Index (ESI) level 4 and 5 complaints (such as an ankle sprain or medication refill), that are staffed primarily by mid-level providers (MLPs), who operate under the supervision of an attending physician. Apart from these fast-track areas, there are no geographical limitations to patient assignments at any of the three sites (such as pods or zones). No resident or trainee physicians rotate at the sites. The physician groups at all three sites are independent of one another.

In each of these EDs, timestamps are automatically recorded by the electronic medical record as physicians assign themselves to patients, and the timestamp data are compiled along with additional aggregated and deidentified patient-level data by LogixHealth, a private company that provides coding, billing and operational analytical services for EDs throughout the USA.

\section{Study protocol}

We abstracted 2 years of patient encounter data from all the ED information systems at three clinical sites (2014-2016). All patient encounters and their related timestamps were provided. All physicians at the three sites were included, and the authors performing the primary analysis were blinded to the physicians' and sites' identities. With the exception of the timestamp data, no other form of patient-identifying information was analysed, and individual timestamps were removed during the course of aggregating data into physician shifts to comply with the American Health Insurance Portability and Accountability Act. A database of physician shifts was created by abstracting new patient assignments and acuity, represented by ESI level. Shifts were subdivided by hour, and all shifts were matched against an external database of physician shift schedules provided by the clinical sites. Aberrant timestamps were identified using a set of algorithms that examined for several specific outliers (assignments to off-shift physicians, patient dispositions prior to assignment and negative lengths of stay).

\section{MEASURES}

Our primary outcome measure was the number of new patient assignments per physician for each hour of the shift. While relative value units (RVUs), which measure estimates of physician effort and resource utilisation, have been used as a supplement or alternative to patient assignments to measure productivity in several prior studies, they are subject to substantial variability between years, particularly for procedures. ${ }^{3}$

\section{Data analysis}

Statistical analysis was performed with Python 3.6, using the SciPy library of packages, ${ }^{6-9}$ with additional model verification run in R. ${ }^{10}$ Our primary outcome measure, new patient assignments per hour, was assessed by multivariable Gaussian regression models using a generalised estimation equation (GEE) method. GEEs are related to traditional linear regression, but are particularly well-suited to data sets in which multiple longitudinal measurements are taken of the same subject, which in this study were groups of consecutive shifts by the same physician. GEEs give robust estimations of population-wide effects, even if the covariance between repeated observations is unknown or incorrectly specified. ${ }^{11} 12$ We defined our model using an exchangeable covariance structure, which assumes equal correlation between terms. Alternative covariance structures, such as an autoregressive (ie, AR(1)), which models a declining correlation over time, were also tested in sensitivity analyses.

The main effect was the shift hour, which was defined in our model as a categorical variable to evaluate differences between individual hours of the shift. The first shift hour was used as the reference value. Thus, the estimate for each subsequent hour demonstrates the hour's expected difference in productivity from the first hour.

Our multivariate analysis included additional a priori independent variables which had been shown to affect productivity from our previous study. These included the number of new available patients per hour (hourly patient arrivals in the ED), the number of concurrent physician providers in a given hour and the median ESI score of the patients seen during each hour. We also included interaction terms between patient arrivals and the individual hours of the shift, which modelled potential changes in the response to arriving patients over subsequent hours. Due to the fact that signed-out patients are generally not transferred on the physician tracking system at the sites investigated, signed-out patients were not counted towards a provider's new patients per hour. This resulted in a final formula of predicted patients per hour $=$ (baseline + hourly adjustment $)+$ (patient arrivals $\times$ (baseline + hourly adjustment $))+$ (doctors working $\times$ coefficient $)+$ (median acuity $\times$ coefficient $)$. We report final parsimonious models as determined by quasi-likelihood score. ${ }^{13}$ A two-sided $\mathrm{P}$ value $<0.05$ was considered statistically significant. 
207,169

Patient Encounters During Study Period

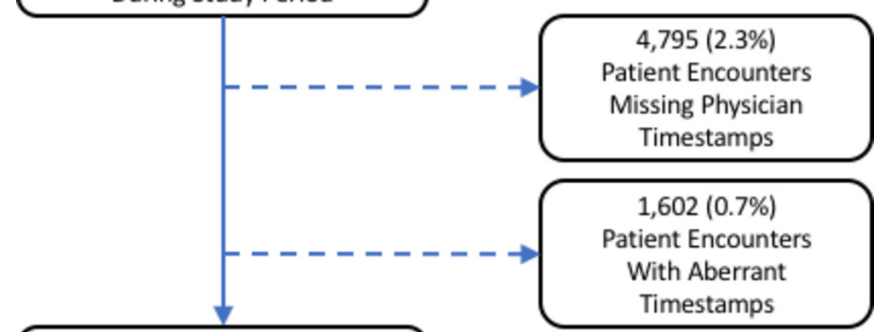

$200,772(97 \%)$

Patient Encounters Evaluated

Patient Encounters Divided by Physician Shifts

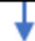

Regression Mode Validation on Physician Shifts

Figure 1 Enrolment and analysis flow chart.

\section{RESULTS}

There were 207169 patient encounters evaluated by 64 attending physicians during the study period. The enrolment process is illustrated in figure 1. Of these encounters, 6397 (3.1\%) had missing data or aberrant timestamps. The missing data constituted 4795 patients who were seen by an MLP for which the encounter was missing an initial physician assignment timestamp. The majority of these encounters (4618) were at site 1 , constituting patients who were seen in the fast-track area and not necessarily seen directly by a physician. The 1602 aberrant timestamps consisted of patient assignments which did not correspond to the correct hours of the physician shift to which it was assigned, indicating that the assigned patient had arrived substantially before the beginning of the shift. The aberrant assignment timestamps were clustered at sites 1 and 2, and had an equivalent distribution in ESI. All encounters with missing data or aberrant timestamps were withheld from the analysis. The final data set for analysis comprised 200772 patient encounters, across 9822 shifts, 4444 from site 1, 2920 from site 2 and 2458 from site 3 . The full details of the clinical sites and physicians are detailed in table 1 .

Across all three sites, the regression model demonstrated a monotonic decrease in productivity with every subsequent hour of the shift (figure 2, tables 2-4). Physicians at sites 1 and 2 had the highest starting productivity at 2.97 patients per hour (95\% CI 2.53 to 3.41) and 2.95 patients per hour (95\% CI 2.47 to 3.43 ), while physicians at site 3 began at 2.17 (95\% CI 2.00 to 2.33). Physicians at all sites demonstrated a significant decline in productivity between the first and second hours of the shift, as well as during the final 2 hours of the shift.

By the midpoint of the shift (hour 4), physicians at all three sites had lost close to half of their initial productivity relative to the first hour (site 1: -1.61 (95\% CI -2.09 to -1.13$)$, site 2 : -1.91 (95\% $\%$ CI -2.37 to -1.44 ), site $3:-0.94$ (95\% CI -1.11 to -0.78$)$ ). By the seventh hour of the shift (1-2hours before arrival of relief),

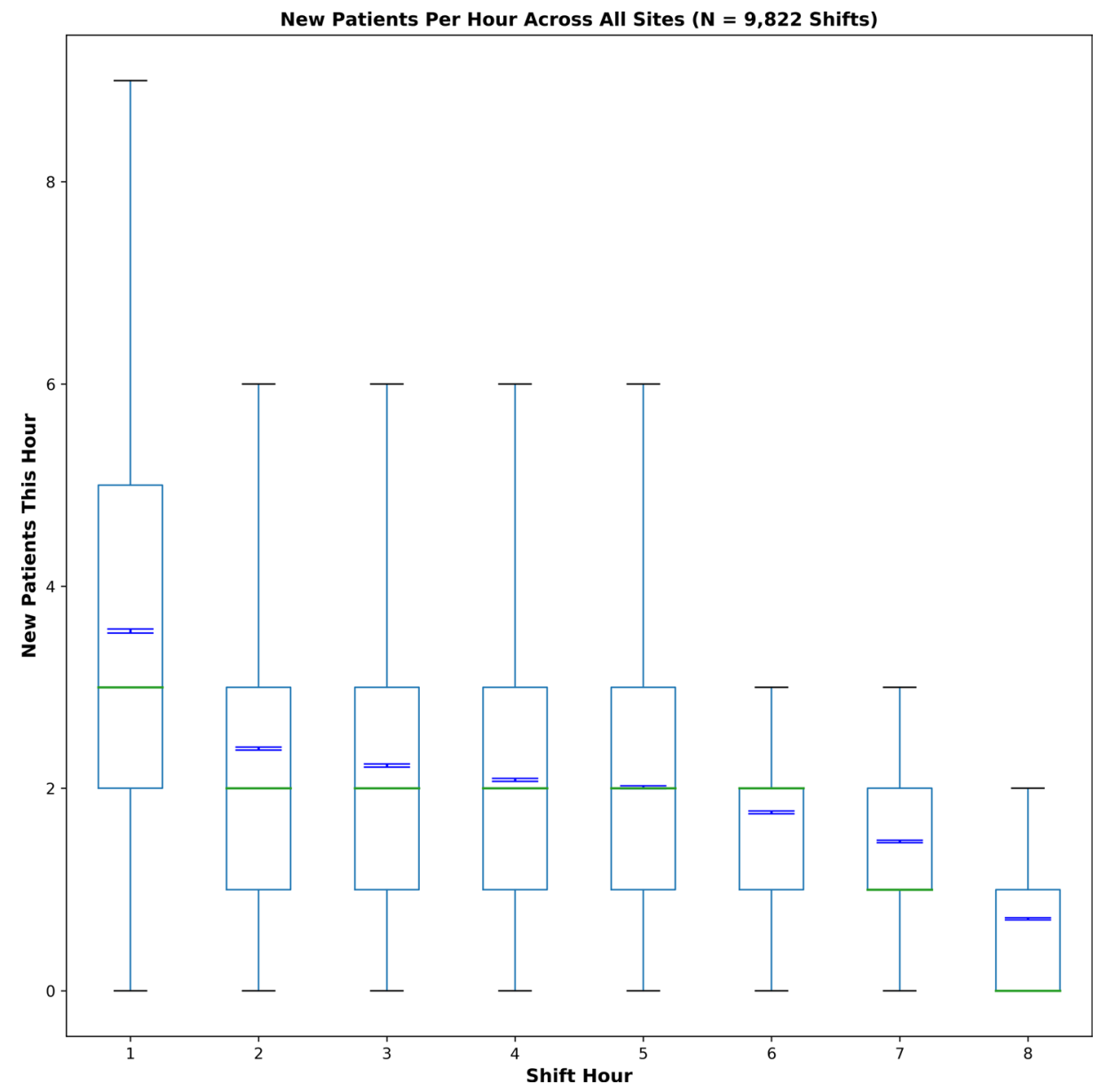

Figure 2 Hourly shift productivity across sites. Median and IQR in green, and means and $95 \% \mathrm{Cl}$ in blue. 
Table 2 Model of attending physician new patients per hour and covariates at site 1

\begin{tabular}{lccl}
\hline & $\begin{array}{l}\text { Estimated new } \\
\text { patients per hour }\end{array}$ & $95 \%$ Cl & P value \\
\hline Hour 1 (intercept) & 2.97 & 2.53 to 3.41 & $<0.01$ \\
\hline Hour 2 & -1.00 & -1.47 to -0.53 & $<0.01$ \\
\hline Hour 3 & -1.40 & -1.87 to -0.92 & $<0.01$ \\
\hline Hour 4 & -1.61 & -2.09 to -1.13 & $<0.01$ \\
\hline Hour 5 & -1.67 & -2.20 to -1.14 & $<0.01$ \\
\hline Hour 6 & -1.69 & -2.20 to -1.16 & $<0.01$ \\
\hline Hour 7 & -2.00 & -2.42 to -1.58 & $<0.01$ \\
\hline Hour 8 & -2.56 & -2.93 to -2.19 & $<0.01$ \\
\hline Arrivals & 0.12 & 0.07 to 0.17 & $<0.01$ \\
\hline Arrivals * hour 2 & -0.04 & -0.11 to 0.03 & $<0.01$ \\
\hline Arrivals * hour 3 & -0.02 & -0.09 to 0.05 & $<0.01$ \\
\hline Arrivals * hour 4 & -0.01 & -0.06 to 0.07 & $<0.01$ \\
\hline Arrivals * hour 5 & -0.01 & -0.07 to 0.09 & $<0.01$ \\
\hline Arrivals * hour 6 & -0.01 & -0.09 to 0.06 & $<0.01$ \\
\hline Arrivals * hour 7 & -0.01 & -0.07 to 0.06 & $<0.01$ \\
\hline Arrivals * hour 8 & -0.08 & -0.13 to -0.03 & $<0.01$ \\
\hline Each additional doctor & -0.37 & -0.42 to -0.32 & $<0.01$ \\
working & & & $<0.01$ \\
\hline Acuity (median ESI) & 0.25 & 0.19 to 0.32 & \\
\hline Asteis ( $)$ denos & &
\end{tabular}

Asterisk $\left({ }^{*}\right)$ denotes interaction terms.

Estimate of new patients per

hour $=(2.97+$ hourly adjustment $)+($ arrivals $\times(0.12+$ hourly adjustment $))+($ doctors working $\times-0.37)+($ median acuity $\times 0.25$ ).

In the first hour of the shift, a physician working alone with five new patient arrivals and a median ESI score of 3 would see an estimate of: $2.97+(5 \times 0.12)+(0 \times$ $-0.37)+(3 \times 0.25)=4.32$ patients.

In the fourth hour of the shift, a physician working alone with five new patient arrivals and a median ESI score of 3 would see an estimate of: $(2.97+-1.61)+(5 \times(0$. $12+-0.01))+(0 \times-0.37)+(3 \times 0.25)=2.66$ patients.

ESI, Emergency Severity Index.

physicians at all three sites fell below one patient per hour. The extent of other hour-to-hour decreases in productivity varied by site, but at all sites the hours during the middle of the shift were associated with less dramatic declines between individual shift hours (tables 1-3).

Additional patient arrivals in the ED were associated with a modest increase in hourly productivity at all sites, but were much more prominent at the two smaller sites (site 1: 0.12 new patients seen per arrival $(95 \% \mathrm{CI} 0.07$ to 0.17 ) vs site $2: 0.37$ (95\% CI 0.31 to 0.43 ) and site $3: 0.42$ (95\% CI 0.35 to 0.48$)$ ). Over the course of the shift, the effect of additional arrivals varied by site, as modelled in the interaction between arrivals and shift hour (tables 2-4). Site 1, at which additional arrivals had only a small effect on individual productivity, saw no significant decrease in this effect until the final hour of the shift. At site 2 , additional patient arrivals were associated with a consistent increase in productivity until the sixth hour of the shift $(-0.11$, $95 \% \mathrm{CI}-0.19$ to -0.04 ), after which the effect of new arrivals declined steadily. At site 3, physician productivity remained sensitive to new arrivals until the fourth hour, when there was a small but statistically significant decline $(-0.10,95 \% \mathrm{CI}-0.17$ to -0.04$)$, and held during the remainder of the shift. By the final hour of the shift, the number of new arrivals required to prompt a physician to see an additional patient diverged widely: site 1-at least seven new arrivals, site 2-at least three new arrivals, and site 3 -at least two new arrivals.

The presence of each additional physician working concurrently was associated with lower productivity at all three sites, most prominently at the lowest volume site (site 1: -0.37 (95\%
Table 3 Model of attending physician new patients per hour and covariates at site 2

\begin{tabular}{lccl}
\hline & $\begin{array}{l}\text { Estimated new } \\
\text { patients per hour }\end{array}$ & $95 \%$ Cl & P value \\
\hline Effect & 2.95 & 2.47 to 3.43 & $<0.01$ \\
\hline Hour 1 (intercept) & -1.35 & -1.71 to -0.99 & $<0.01$ \\
\hline Hour 3 & -1.62 & -2.04 to -1.20 & $<0.01$ \\
\hline Hour 4 & -1.91 & -2.37 to -1.44 & $<0.01$ \\
\hline Hour 5 & -2.07 & -2.54 to -1.61 & $<0.01$ \\
\hline Hour 6 & -1.93 & -2.40 to -1.49 & $<0.01$ \\
\hline Hour 7 & -2.12 & -2.54 to -1.72 & $<0.01$ \\
\hline Hour 8 & -2.69 & -3.03 to -2.34 & $<0.01$ \\
\hline Arrivals & 0.37 & 0.31 to 0.43 & $<0.01$ \\
\hline Arrivals * hour 2 & -0.01 & -0.06 to 0.03 & $<0.01$ \\
\hline Arrivals * hour 3 & 0.00 & -0.01 to 0.09 & $<0.01$ \\
\hline Arrivals * hour 4 & 0.01 & -0.04 to 0.06 & $<0.01$ \\
\hline Arrivals * hour 5 & 0.02 & -0.05 to 0.09 & $<0.01$ \\
\hline Arrivals * hour 6 & -0.11 & -0.19 to -0.04 & $<0.01$ \\
\hline Arrivals * hour 7 & -0.14 & -0.22 to -0.07 & $<0.01$ \\
\hline Arrivals * hour 8 & -0.23 & -0.29 to -0.17 & $<0.01$ \\
\hline Each additional doctor & -0.27 & -0.34 to -0.21 & $<0.01$ \\
\hline working & & & $<0.01$ \\
\hline Acuity (median ESI) & 0.12 & 0.05 to 0.20 & \\
\hline Astrisk ( ) den & &
\end{tabular}

Asterisk $\left({ }^{*}\right)$ denotes interaction terms.

Estimate of new patients per

hour $=(2.95$-hourly adjustment $)+($ arrivals $\times(0.37+$ hourly adjustment $))+($ doctors working $\times-0.27)+($ median acuity $\times 0.25)$.

In the first hour of the shift, a physician working alone with five new patient arrivals and a median ESI score of 3 would see an estimate of: $2.95+(5 \times 0.37)+(0 \times$ $-0.27)+(3 \times 0.25)=5.55$ patients.

In the fourth hour of the shift, a physician working alone with five new patient arrivals and a median ESI score of 3 would see an estimate of: $(2.95+-1.91)+(5 \times(0$. $37+0.01))+(0 \times-0.27)+(3 \times 0.25)=3.69$ patients.

ESI, Emergency Severity Index.

CI -0.42 to -0.32$)$, site $2:-0.27(95 \%$ CI -0.34 to -0.21$)$, site 3: $-0.43(95 \% \mathrm{CI}-0.50$ to -0.36$))$. Lower median hourly acuity was associated with a mild increase in productivity for every point of less acute ESI score (higher ESI score), most prominently at site $1(0.25,95 \%$ CI 0.19 to 0.32$)$.

\section{DISCUSSION}

'How many patients should I be seeing?' is a fundamental professional question for the practising emergency physician, and 'how should physicians be scheduled?' has bedevilled many an administrator. While there exists a substantial body of operational research literature in emergency medicine, investigations of the productivity of individual emergency physicians in the community hospital setting have been limited. The majority of prior studies reporting measures of attending physician productivity have reported shift-level productivity measures (split between average patients per hour and RVUs per hour) within an academic rather than community setting, limiting their generalisability. ${ }^{14-21}$ Often, measures of individual productivity have been reported as a measure of specific interventions, such as the introduction of scribes, residents or informatics tools. ${ }^{162022-24}$ To date, no study has examined the pattern of attending physicians' accrual of new patients over the course of a shift.

This study demonstrates that attending physicians' productivity (as measured by new patients seen per hour) decreases predictably over the course of a shift, even after adjusting for the number of new patient arrivals per hour, the number of physicians working concurrently and acuity. This finding is similar to that of our previous study of resident physicians. ${ }^{3}$ Of note, attending physicians in our 
Table 4 Model of attending physician new patients per hour and covariates at site 3

\begin{tabular}{lccl}
\hline Effect & $\begin{array}{l}\text { Estimated new } \\
\text { patients per hour }\end{array}$ & $95 \% \mathrm{Cl}$ & P value \\
\hline Hour 1 (intercept) & 2.17 & 2.00 to 2.33 & $<0.01$ \\
\hline Hour 2 & -0.79 & -1.00 to -0.57 & $<0.01$ \\
\hline Hour 3 & -1.05 & -1.22 to -0.88 & $<0.01$ \\
\hline Hour 4 & -0.94 & -1.11 to -0.78 & $<0.01$ \\
\hline Hour 5 & -1.01 & -1.16 to -0.86 & $<0.01$ \\
\hline Hour 6 & -1.06 & -1.21 to -0.91 & $<0.01$ \\
\hline Hour 7 & -1.21 & -1.31 to -1.10 & $<0.01$ \\
\hline Hour 8 & -1.43 & -1.56 to -1.31 & $<0.01$ \\
\hline Arrivals & 0.42 & 0.35 to 0.48 & $<0.01$ \\
\hline Arrivals * hour 2 & -0.08 & -0.16 to 0.01 & $<0.01$ \\
\hline Arrivals * hour 3 & -0.06 & -0.12 to 0.00 & $<0.01$ \\
\hline Arrivals * hour 4 & -0.10 & -0.17 to -0.04 & $<0.01$ \\
\hline Arrivals * hour 5 & -0.09 & -0.14 to -0.03 & $<0.01$ \\
\hline Arrivals * hour 6 & -0.09 & -0.16 to -0.02 & $<0.01$ \\
\hline Arrivals * hour 7 & -0.08 & -0.13 to -0.03 & $<0.01$ \\
\hline Arrivals * hour 8 & -0.09 & -0.16 to -0.03 & $<0.01$ \\
\hline Each additional doctor & -0.43 & -0.50 to -0.36 & $<0.01$ \\
\hline working & & & $<0.01$ \\
\hline Acuity (median ESI) & 0.06 & 0.04 to 0.08 & \\
\hline Asterisk ( ) dentes in & & \\
\hline
\end{tabular}

Asterisk $\left({ }^{*}\right)$ denotes interaction terms.

Estimate of new patients per

hour $=(2.17+$ hourly adjustment $)+($ arrivals $\times(0.42+$ hourly adjustment $)+($ doctors working $\times-0.43)+($ median acuity $\times 0.06)$.

In the first hour of the shift, a physician working alone with five new patient arrivals and a median ESI score of 3 would see an estimate of:

$2.17+(5 \times 0.42)+(0 \times-0.43)+(3 \times 0.06)=4.45$ patients.

In the fourth hour of the shift, a physician working alone with five new patient arrivals and a median ESI score of 3 would see an estimate of: $(2.17+-0.94)+(5 \times(0$. $42+-0.10))+(0 x-0.43)+(3 \times 0.06)=3.01$ patients.

ESI, Emergency Severity Index.

study demonstrate more gradual declines in productivity after the first hours of their shifts than do residents. This may be due to the impact of further experience after residency, or necessity in the face of a busy ED. Accordingly, viewing physician productivity as a simple average of patients per hour relative to the total duration of the shift will tend to significantly underestimate a physician's capacity to see new patients at the beginning of the shift, and overestimate a physician's capacity at the end of a shift, illustrated in the substantial variations between individual shift hours (tables 1-3).

The most straightforward explanation for this decline in productivity over the course of the shift is mechanistic. At the beginning of a shift, an emergency physician is free to see as many patients as she wishes. However, unless a patient is ready for immediate discharge after a history and physical, the physician will need to order diagnostic testing or therapeutic measures, which may take a considerable amount of time to complete. While this workup is pending, the physician can see additional patients, but at some point the need to address incoming results for her existing patients will limit her ability to see new patients. The pattern of relatively rapid accrual of new patients in the first hours of the shift, followed by a more gradual decline in the rate at which physicians see patients later in the shift, may reflect physicians continuing to work at a steady state. This burden may be further compounded by physical and cognitive fatigue, as emergency physicians' shift schedules are often out of alignment with circadian rhythms.

Among the clinical sites that our study examined, there was considerable variability in the total number of patients that attending physicians saw during a shift. This likely reflects the availability of patients to be seen relative to the number of physicians working at a time. Site 2 , where physicians were most productive per shift, had the greatest daily volume of patients per physician. Similarly, physicians at sites 2 and 3, which had longer periods of single coverage, saw more substantial gains in productivity with the arrival of additional patients than at site 1 , where arrivals were more likely to be spread among a greater number of physicians. Conversely, the pronounced negative association between acuity and productivity at site 1 relative to the other sites may reflect the availability of other physicianswhen backup is available (or arriving imminently), a physician may be able to devote more time to acute patients.

It is important to note that physicians' productivity exists within a wider context. Our study did not examine potential bottlenecks to patient availability, such as periods of increased crowding and decreased availability of inpatient beds, which could cause a substantial disconnect between patient arrivals and physicians' ability to see them. Additionally, there exist additional dimensions to physician productivity, such as the time to make a diagnosis and decide a patient's disposition, and the potentially additive effects of interruptions from managing multiple patients, ${ }^{23}$ which our study does not measure directly.

\section{Limitations}

Our study was conducted at three American suburban community EDs that do not provide tertiary care and are not trauma centres. While the sites have separate groups of physicians, we cannot be certain that cultural effects did not influence productivity patterns. Similarly, although our productivity patterns were similar at all three sites, we cannot be certain that these patterns would persist at sites with much higher volumes or with different patterns of acuity. For example, sites with a lower proportion of acute patients might allow physicians to maintain a more consistent rate of throughput. Both of these factors impact the generalisability of our results. We did not include the number of patients seen by MLPs as an explicit covariate, as the majority of patients seen by MLPs at both site 1 and site 3 are seen under physician supervision, but are not specifically evaluated by the physician. Our analysis did not compare the effects of differing times of day between shifts. Our analysis did not include hospital-level characteristics of efficiency or capacity, such as the time to be transported to an inpatient bed or nurse staffing ratios, although they have previously been identified as factors affecting ED throughput and crowding. We also did not examine sign-out burden or procedures as covariates.

\section{CONCLUSIONS}

Our study demonstrates that attending physicians see a decreasing number of new patients over the course of a shift, but remain responsive to new patient arrivals until late in their shift. This suggests that physician shifts should be aligned to start near times of high volume to maximise physicians' capacity to see new patients, and responsiveness to surges in volume, while physician shifts should be staggered to avoid competing for the same pool of patients. The effects of individual productivity on overall throughput, patient safety and physician burnout remain open questions, towards which we hope our findings spur further investigation.

Acknowledgements This work was conducted with support from Harvard Catalys | The Harvard Clinical and Translational Science Center (National Center for Research Resources and the National Center for Advancing Translational Sciences, National Institutes of Health Award UL1 TR001102) and financial contributions from Harvard University and its affiliated academic healthcare centers. 
Contributors JWJ, SD and LDS conceived of the study. SD and OL performed initial data gathering and aggregation. JWJ performed the initial data analysis and modelling, EHW performed further analysis and model verification. JWJ drafted the manuscript, and all authors contributed substantially to its revision. JWJ takes responsibility for the paper as a whole.

Funding This study was funded by Harvard Medical School (10.13039/100006691). Additional support was provided by the Eleanor and Miles Shore 50th Anniversary Fellowship Program for Scholars in Medicine at Harvard Medical School.

Disclaimer The content is solely the responsibility of the authors and does not necessarily represent the official views of Harvard Catalyst, Harvard University and its affiliated academic healthcare centres, or the National Institutes of Health.

Competing interests SD and OL are employees of LogixHealth, which provides billing, coding and analytics services for EDs throughout the USA.

Patient consent Not required.

Ethics approval The study was granted an exemption to examine operational and productivity metrics by the institutional review board of the academic hospital (Beth Israel Deaconess Medical Center IRB) affiliated with two of the sites (none of the hospital sites have their own institutional review board).

Provenance and peer review Not commissioned; externally peer reviewed.

Open Access This is an Open Access article distributed in accordance with the Creative Commons Attribution Non Commercial (CC BY-NC 4.0) license, which permits others to distribute, remix, adapt, build upon this work non-commercially, and license their derivative works on different terms, provided the original work is properly cited and the use is non-commercial. See: http://creativecommons.org/ licenses/by-nc/4.0/

(c) Article author(s) (or their employer(s) unless otherwise stated in the text of the article) 2018. All rights reserved. No commercial use is permitted unless otherwise expressly granted.

\section{REFERENCES}

1 DeBehnke $D, O^{\prime} B$ rien $S$, Leschke R. Emergency medicine resident work productivity in an academic emergency department. Acad Emerg Med 2000;7:90-2.

2 Hamden K, Jeanmonod D, Gualtieri D, et al. Comparison of resident and mid-level provider productivity in a high-acuity emergency department setting. Emerg Med J 2014;31:216-9.

3 Joseph JW, Henning DJ, Strouse CS, et al. Modeling hourly resident productivity in the emergency department. Ann Emerg Med 2017;70:185-90.

4 Improving patient flow and reducing emergency department crowding: a guide for hospitals. https://www.ahrq.gov/research/findings/final-reports/ptflow/section3.html (accessed 8 Mar 2017)
5 Handel D, Epstein S, Khare R, et al. Interventions to improve the timeliness of emergency care: Blackwell Publishing Ltd, 2011:1295-302.

6 Oliphant TE. Python for scientific computing. Comput Sci Eng 2007;9:10-20.

7 McKinney W. Data structures for statistical computing in python. Proceedings of the 9th Python in Science Conference, 2010:51-6.

8 Hunter JD. Matplotlib: a 2D graphics environment. Comput Sci Eng 2007;9:90-5.

9 Seabold S, Perktold J. Statsmodels: econometric and statistical modeling with python. Proceedings of the 9th Python in Science Conference, 2010:57-61.

10 Halekoh U, Højsgaard S, Yan J. The $R$ package geepack for generalized estimating equations. J Stat Softw 2006;15.

11 Zeger SL, Liang KY. Longitudinal data analysis for discrete and continuous outcomes. Biometrics 1986;42:121-30.

12 Hubbard AE, Ahern J, Fleischer NL, et al. To GEE or not to GEE: comparing population average and mixed models for estimating the associations between neighborhood risk factors and health. Epidemiology 2010;21:467-74.

13 Pan W. Akaike's information criterion in generalized estimating equations. Biometrics 2001;57:120-5.

14 Kelly SP, Shapiro N, Woodruff M, et al. The effects of clinical workload on teaching in the emergency department. Acad Emerg Med 2007;14:526-31.

15 Berger TJ, Ander DS, Terrell ML, et al. The impact of the demand for clinical productivity on student teaching in academic emergency departments. Acad Emerg Med 2004;11:1364-7.

16 France DJ, Levin S, Hemphill R, et al. Emergency physicians' behaviors and workload in the presence of an electronic whiteboard. Int J Med Inform 2005;74:827-37.

17 Begaz T, Decker MC, Treat R, et al. No relationship between measures of clinical efficiency and teaching effectiveness for emergency medicine faculty. Emerg Med J 2011;28.

18 Hemphill RR, Heavrin BS, Lesnick J, et al. Those who can, do and they teach too: faculty clinical productivity and teaching. West J Emerg Med 2011;12:254-7.

19 Clyne B, Smith JL, Napoli AM. Influence of clinical experience and productivity on emergency medicine faculty teaching scores. J Grad Med Educ 2012;4:434-7.

20 Akbar S, Radeos MS, Yang A, et al. 403: Impact of emergency medicine residents on attending physician productivity: a case-control study. Ann Emerg Med 2007;50:S127.

21 Clinkscales JD, Fesmire FM, Hennings JR, et al. The effect of emergency medicine residents on clinical efficiency and staffing requirements. Acad Emerg Med 2016;23:78-82.

22 Arya R, Salovich DM, Ohman-Strickland P, et al. Impact of scribes on performance indicators in the emergency department. Acad Emerg Med 2010;17:490-4.

23 Ward MJ, Landman AB, Case K, et al. The effect of electronic health record implementation on community emergency department operational measures of performance. Ann Emerg Med 2014;63:723-30.

24 Walker KJ, Dunlop W, Liew D, et al. An economic evaluation of the costs of training a medical scribe to work in Emergency Medicine. Emerg Med J 2016;33:865-9. 